\title{
MOST SPACE TELESCOPE PHOTOMETRY OF THE 2010 JANUARY TRANSIT OF EXTRASOLAR PLANET HD80606b
}

\author{
Jessica E. Roberts ${ }^{1}$, Jason W. Barnes ${ }^{2}$, Jason F. Rowe ${ }^{3}$, and Jonathan J. Fortney ${ }^{4}$ \\ ${ }^{1}$ Department of Physics and Astronomy, San Jose State University, Room 148, San Jose, CA 95192-0106, USA; jessica.roberts@ @jsu.edu \\ ${ }^{2}$ University of Idaho, Department of Physics, Campus Box 440903, Moscow, ID 83844-0903, USA \\ 3 NASA Ames Research Center, M/S 244-30, Moffett Field, CA 94035, USA \\ ${ }^{4}$ Department of Astronomy and Astrophysics, University of California, Santa Cruz, CA 95064, USA \\ Received 2011 July 27; accepted 2012 November 7; published 2012 December 14
}

\begin{abstract}
We present observations of the full 2010 January transit of HD80606b from the Canadian microsatellite, Microvariability and Oscillations of Stars. By employing a space-based telescope, we monitor the entire transit, thus limiting systematic errors that result from ground observations. We determine measurements for the planetary radius $\left(R_{p}=0.987 \pm 0.061 R_{\mathrm{Jup}}\right)$ and inclination $(i=89.283 \pm 0.024)$ by constraining our fits with the observed parameters of different groups. Our measured mid-transit time of $2455210.6449 \pm 0.0034$ HJD is consistent with the 2010 Spitzer results and is 20 minutes earlier than predicted by groups who observed the 2009 June transit.
\end{abstract}

Key words: planets and satellites: individual (HD80606b) - techniques: photometric

Online-only material: color figures

\section{INTRODUCTION}

With its unusually high eccentricity of $e=0.93$ and long orbital period of 111 days, HD80606b challenges our understanding of exoplanets. This planet was discovered by Naef et al. (2001) via radial velocity techniques in which they determined a minimum planetary mass of $3.9 M_{\text {Jup }}$. The occultation of HD80606b was observed with the Spitzer Space Telescope eight years later, indicating that the inclination of the planet is greater than $89^{\circ}$ (Laughlin et al. 2009). The existence of an occultation indicated that there was a $15 \%$ chance that this planet would also have a visible primary transit.

Despite the low probability, several independent groups detected the 2009 February primary transit from groundbased observatories (Fossey et al. 2009; Garcia-Melendo \& McCullough 2009; Hidas et al. 2010; Pont et al. 2009; Moutou et al. 2009). The full transit was later observed by Winn et al. (2009) who used multiple ground-based observatories to sew together complete coverage.

Winn et al. (2009) also confirmed a spin-orbit misalignment of $53^{\circ}$ that was first predicted by Moutou et al. (2009). It is possible that the high eccentricity and tilted orbit of HD80606b are the result of the Kozai mechanism induced by the system's binary stellar companion HD80607 (Wu \& Murray 2003).

Since the $12 \mathrm{hr}$ duration of HD80606b's transit presents a difficulty for accurate ground-based observations, Hébrard et al. (2010) employed the combination of Spitzer photometry and SOPHIE spectroscopy to continuously observe the full 2010 January transit. Hébrard et al. (2010) discovered their midtransit time to be approximately 24 minutes earlier than the value predicted by Winn et al. (2009). In addition, Hébrard et al. (2010) found their $R_{p} / R_{*}$ value differed by $3 \sigma$ compared to the results obtained by both Pont et al. (2009) and Winn et al. (2009).

Shporer et al. (2010) launched a multiple-site ground-based observation of the same transit in an attempt to confirm the Hébrard et al. (2010) timing discrepancy. Their mid-transit time was 12 minutes earlier than predicted and within $1.3 \sigma$ of Hébrard et al. (2010).
We observe the 2010 January transit of HD80606b with the Microvariability and Oscillations of Stars (MOST) earthorbiting optical telescope with the goals of: (1) determining a mid-transit time using both Winn et al. (2009) and Hébrard et al. (2010) orbital parameters and (2) deriving an independent set of results for the planetary radius, inclination, and mid-transit time. Our observations by the MOST telescope are described in Section 2, our light curve modeling is detailed in Section 3, and we present and discuss our results to both goals mentioned above in Section 4. We conclude this paper in Section 5.

\section{OBSERVATIONS}

The MOST is a microsatellite with a $15 \mathrm{~cm}$ aperture photometer designed for asteroseismology, but is well suited to observe short-period exoplanets around solar-type stars (Rowe et al. 2006). The satellite employs a broadband visible filter centered at $525 \mathrm{~nm}$. MOST is in a low polar, sun-synchronous orbit that permits for a continuous viewing zone $(\mathrm{CVZ})$ of stars between the declination of $-19^{\circ}$ and $+36^{\circ}$. HD80606b, however, has a declination of $+50^{\circ}$ placing it outside of the CVZ. Therefore, we experience short blackouts in our data as Earth passes in between MOST and HD80606. We are unable to observe the 2010 January 8 secondary eclipse of HD80606b due to an inconvenient instrumentation issue that led to a $12 \mathrm{hr}$ gap in our data.

We observe HD80606b starting from 24548677.97302083 HJD and continuing to 2454877.85480324 HJD (2010 January 7-January 19) with an exposure time of $80.40 \mathrm{~s}$ integrations. Since HD80606 is a magnitude 9.06 star, we use the direct imaging mode of the CCD and employ simple aperture photometry with a field of view of $20 \times 20$ pixels. We capture images of both HD80606 and HD80607, but we are able to constrain HD80606 within a 3 pixel radius compared to the binary stars' separation of 10 pixels.

Stray light for MOST varies from 100 ADU to 1500 ADU as it passes over the polar caps and day/night boundary of Earth. We remove data that have a background level above 800 ADU (about 100,000 points). At this lower level of stray light, our photometric error is equal to the expected Poisson noise 


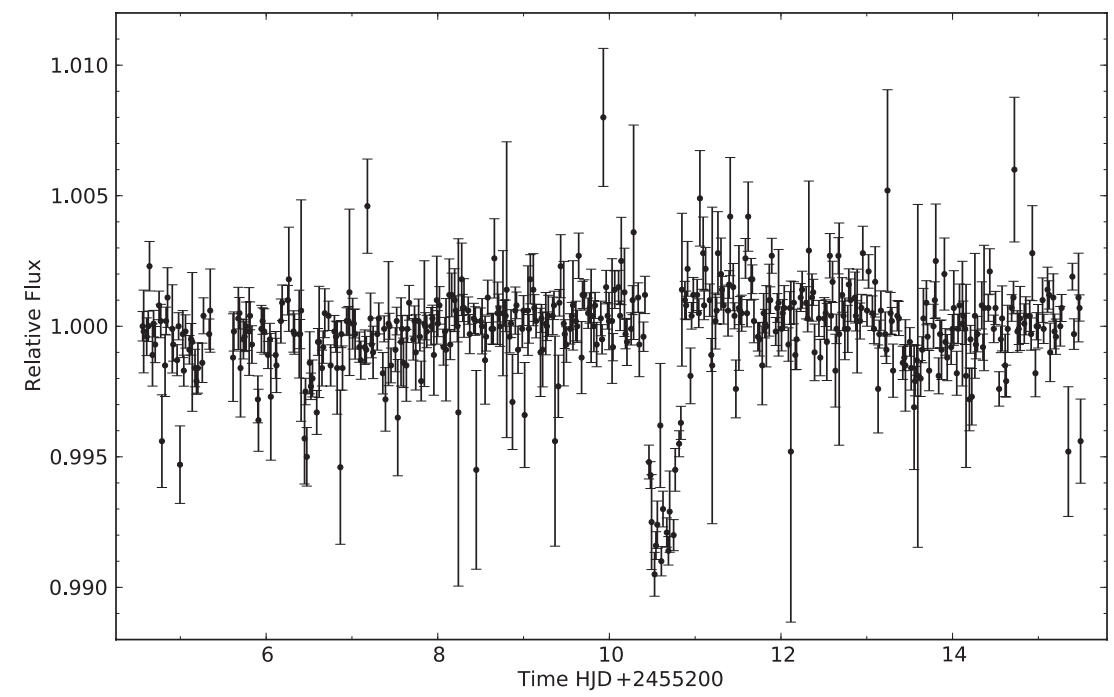

Figure 1. Here we show the MOST photometry full data set, including errors. The photometric data are binned to one point every 15 minutes.

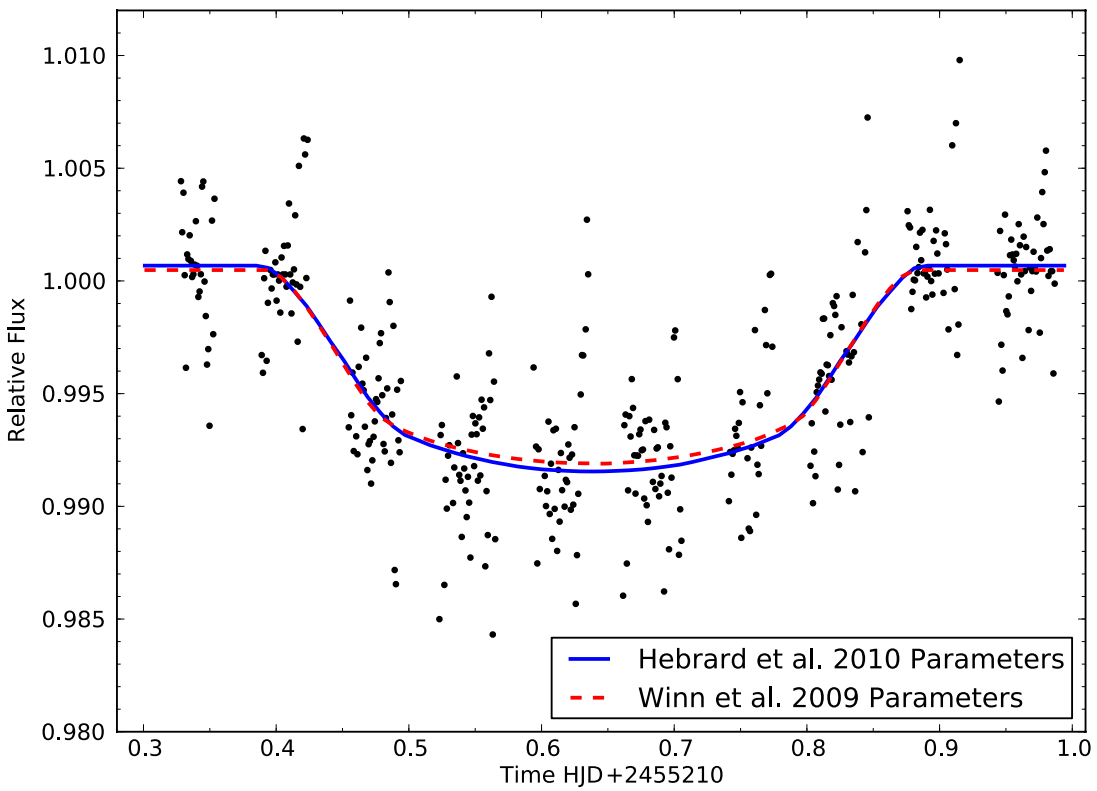

Figure 2. Unbinned close-up view of the MOST transit data. The two lines represent our two best fits. The blue line is our best fits constrained by Hébrard et al. (2010) parameters, whereas the red dashed line is the best fit constrained by Winn et al. (2009) parameters.

(A color version of this figure is available in the online journal.)

limit. For a detailed procedure of standard observation and data reduction of MOST data, see Rowe et al. (2006).

Figure 1 shows our full photometric time series, with points binned every 15 minutes for clarity. By binning the data the gaps are not as apparent; however, we fit the full unbinned transit (Section 3). Due to the binning effect, the transit in Figure 1 appears asymmetric. A close-up view of the unbinned transit in Figure 2 demonstrates that within the error of our measurement the transit is symmetric and we are not forced to make necessary modifications for an asymmetric effect. The large gap occurring at 24552005.5 is due to the instrumentational error discussed above.

\section{LIGHT CURVE MODELING}

We use the fitting process described in Barnes \& Fortney (2003). With high eccentricity, the transit duration is correlated to the inclination and argument of periapsis of the planet's orbit.
The transit ingress and egress times also experience a several minute asymmetry, with the ingress duration shorter than the egress in the case of HD80606b. We account for these variations by explicitly incorporating orbital eccentricity in to our code, as detailed in Barnes (2007).

In this routine, we do not account for the impact parameter $b$ as this parameter is directly related to the inclination in an eccentric orbit by

$$
b=\left(\frac{a}{R_{*}}\right)\left(\frac{1-e^{2}}{1+e * \sin (\omega)}\right) \cos (i) .
$$

By fitting for the inclination alone, we accelerate the fitting algorithm.

We fit the out-of-transit flux dynamically in our model instead of assuming a value. Out-of-transit points that fall more than $3 \sigma$ outside of this photometric baseline are removed. Our bestfit transit model is determined by minimizing $\chi^{2}$ through a 


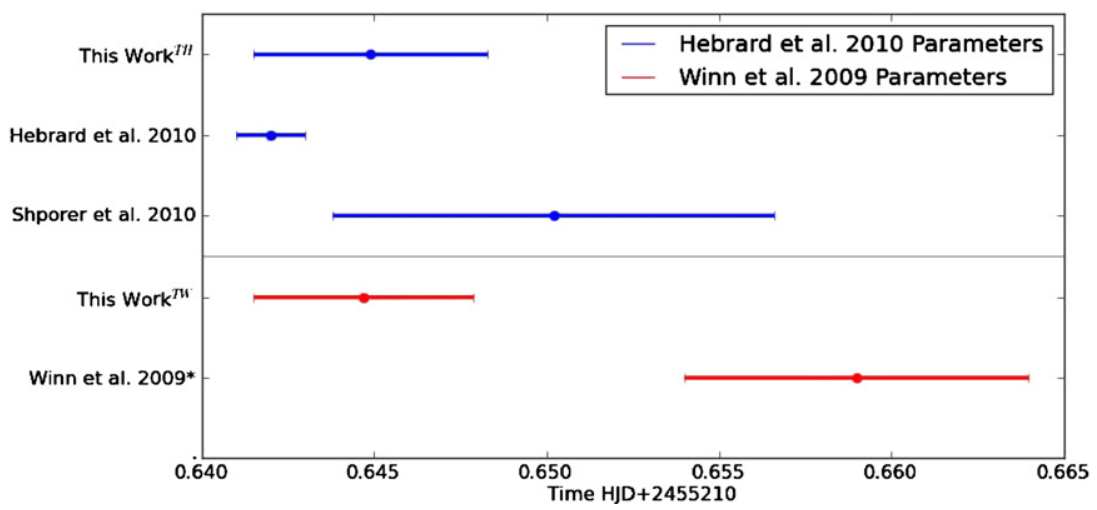

Figure 3. Five different mid-transit timing results from different groups with errors included. The top three results in blue were calculated using Hébrard et al. (2010) parameters. The bottom two results in red were calculated using Winn et al. (2009) parameters.

(A color version of this figure is available in the online journal.)

Levenberg-Marquardt fitting algorithm adapted from Press et al. (1992).

As our data are not precise enough to fit for the limb-darkening parameters combined with our other fitted parameters, we hold both coefficients constant. Our limb-darkening parameters are established by using the defined MOST quadratic parameters of HD80606 with a $T_{\text {eff }}=5645 \mathrm{~K}$ and $\log g=4.50$ (Naef et al. 2001). These coefficients are manipulated to fit our model by using the limb-darkening method highlighted in Brown et al. (2001). Our first coefficient, designated as $c_{1}=$ 0.742 , represents the magnitude of darkening while our second coefficient, $c_{2}=0.458$, accounts for the curvature of the transit (Barnes \& Fortney 2003).

We do not account for any other systematic effects or correlated noise. Therefore, we assume all errors to be random with a Gaussian distribution. See Barnes \& Fortney (2003) and Barnes (2007) for additional details regarding our model.

We execute two different model fits: the first model is used to verify the mid-transit time reported by Hébrard et al. (2010) and a second to determine our own independent parameters. In our effort to reproduce the Hébrard et al. (2010) mid-transit time, we hold all their parameters constant and fit solely for the baseline flux and mid-transit time. We repeat this procedure using Winn et al. (2009) parameters for comparison to their predicted mid-transit time. For clarity, we will denote our timing results with either a superscript TH for Hébrard et al. (2010) and TW for Winn et al. (2009) when discussing out results in Section 4.1.

In establishing our independent best-fit parameters, we determine that our data are only adequate to fit for four parameters: planetary radius $\left(R_{p}\right)$, mid-transit time $\left(T_{\text {mid }}\right)$, inclination $(i)$, and the baseline flux. We constrain our two fits with the parameters from Hébrard et al. (2010) and Winn et al. (2009) for stellar radius, stellar mass, period, eccentricity, argument of periapsis, and semimajor axis. We analyze these results in Section 4.2.

\section{DISCUSSION}

\subsection{Mid-transit Time Fit Only}

In this section, we discuss our mid-transit time results of HD80606b with a goal of verifying the early mid-transit time discovered by Hébrard et al. (2010). In order to measure a midtransit time appropriate for comparison, we hold Hébrard et al. (2010) orbital, stellar, and planetary parameters constant and fit only for the mid-transit time and baseline flux.
Table 1

Our Mid-transit Times for the 2010 January Transit

\begin{tabular}{lcc}
\hline \hline & \multicolumn{1}{c}{ Mid-transit Time } & $\chi_{\mathrm{r}}^{2}$ \\
\hline MOST (This Work) $^{\mathrm{TH}}$ & $2455210.6449 \pm 0.0034$ HJD & 1.11735 \\
\hline Spitzer (Hébrard et al. 2010) $^{\text {Ground-based (Shporer et al. 2010) }}$ & $2455210.6420 \pm 0.0010$ HJD & \\
\hline MOST (This Work) & $245210.6502 \pm 0.0064$ HJD & \\
\hline Ground-based (Winn et al. 2009 $)$ & $2455210.6590 \pm 0.0050$ HJD & \\
\hline
\end{tabular}

Notes. "MOST $T^{\mathrm{TH}}$ " designates mid-transit time using parameters determined by Hébrard et al. (2010). "MOST ${ }^{\mathrm{TW}}$ " represents the mid-transit time determined with Winn et al. (2009) parameters. $\chi_{r}^{2}$ shows the reduced $\chi^{2}$ of our best fit. a Predicted.

Our resulting mid-transit time (denoted with the superscript TH) is 20 minutes earlier than predicted by Winn et al. (2009). For comparison, Hébrard et al. (2010) found a mid-transit time 24 minutes (17 $\sigma$ difference) earlier than the value predicted by Winn et al. (2009).

Orbital parameters have a direct effect on mid-transit times, especially in a highly eccentric orbit. Since Hébrard et al. (2010) determined a separate set of orbital parameters compared to Winn et al. (2009), we also fit the MOST light curve using the latter's parameters for the mid-transit time. Even with the different orbital parameters, we determine a mid-transit time (denoted with the superscript TW) 20 minutes early as well.

We conclude that the difference in mid-transit times between the two groups is not dependent on their varying orbital parameters. Furthermore, we determine that our results have less than a $1 \sigma$ discrepancy from the mid-transit time determined by Hébrard et al. (2010). A visual comparison of each midtransit time with uncertainties is displayed in Figure 3. We also highlight the mid-transit timing results from an independent ground-based group, Shporer et al. (2010), in Figure 3 who observed the 2010 January transit. Our overall timing results are also listed in Table 1.

Our mid-transit time agrees with the Hébrard et al. (2010) result to a less than $1 \sigma$ difference. We also find that our midtransit time has $1.6 \sigma$ difference with the result determined by Shporer et al. (2010) and a greater than $3 \sigma$ difference from the predicted transit time of Winn et al. (2009). In fact, the only result that agrees with the Winn et al. (2009) predicted time (within 1 $\sigma$ ) is Shporer et al. (2010). They note, however, that due to the difficulty of ground-based observations, the uncertainty 
Table 2

The Two Groups of Parameters That Were Held Constant While Fitting

\begin{tabular}{|c|c|c|c|c|c|c|}
\hline & \multicolumn{6}{|c|}{ Constant Parameters } \\
\hline & $R_{*}$ & $M_{*}$ & $P$ & $a$ & $e$ & $\omega$ \\
\hline Hébrard et al. (2010) Parameters ${ }^{\mathrm{FH}}$ & $1.007 R_{\text {Sun }}$ & $1.01 M_{\text {Sun }}$ & 111.4367 days & $0.455 \mathrm{AU}$ & 0.9330 & 300.77 \\
\hline Winn et al. (2009) Parameters ${ }^{F W}$ & $0.968 R_{\text {Sun }}$ & $1.05 M_{\text {Sun }}$ & 111.4374 days & $0.4614 \mathrm{AU}$ & 0.93286 & 300.83 \\
\hline
\end{tabular}

Notes. Fitting runs using the Hébrard et al. (2010) parameters are denoted with ${ }^{\mathrm{FH}}$ while best-fit results calculated with the Winn et al. (2009) parameters are designated with $\mathrm{FW}$.

Table 3

A Comparison of Fitted Parameters

\begin{tabular}{|c|c|c|c|c|c|}
\hline & \multicolumn{5}{|c|}{ Fitted Parameters } \\
\hline & $R_{P}$ & $R_{P} / R_{*}$ & $i$ & Mid-transit Time & $\chi_{r}^{2}$ \\
\hline This Work $^{\mathrm{FH}}$ & $0.987_{-0.061}^{+0.013} R_{\text {Jup }}$ & $0.1007_{-0.0062}^{+0.0013}$ & $89.283 \pm 0.024$ & $2455210.6461 \pm 0.00781 \mathrm{HJD}$ & 1.01389 \\
\hline Hébrard et al. (2010) & $0.981 \pm 0.023 R_{\text {Jup }}$ & $0.1001 \pm 0.006$ & $89.269 \pm 0.018$ & $2455210.6420 \pm 0.0010 \mathrm{HJD}$ & \\
\hline 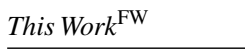 & $0.905 \pm 0.032 R_{\mathrm{Jup}}$ & $0.0961 \pm 0.0034$ & $89.346 \pm 0.022$ & $2455210.6439 \pm 0.00773$ HJD & 1.10136 \\
\hline Winn et al. (2009) & $0.974 \pm 0.030 R_{\text {Jup }}$ & $0.1033 \pm 0.0011$ & $89.324 \pm 0.029$ & $2455210.6590 \pm 0.0050 \mathrm{HJD}^{\mathrm{a}}$ & \\
\hline
\end{tabular}

Notes. This Work ${ }^{\mathrm{FH}}$ represents best fit values using Hébrard et al. (2010) constraints. This Work ${ }^{\mathrm{FW}}$ shows best-fit values using Winn et al. (2009) parameters. The variables determined by the two other groups are shown for comparison. $\chi_{r}{ }^{2}$ is shown for a statistical comparison of the two fits.

${ }^{a}$ Predicted result.

in their measurement is large. Shporer et al. (2010) still find a mid-transit time of 12 minutes earlier than Winn et al. (2009) predicted, a large discrepancy regardless of the uncertainty.

One possible explanation for the large difference between the mid-transit times of this work and Winn et al. (2009) is the possibility of a perturbing body in the system creating a transit timing variation (TTV). However, Naef et al. (2001) show that if there is another body perturbing this system, it is not detectable via radial velocity measurements. Wu \& Murray (2003) also argue that while another planet may have formed around HD80606, no Jupiter-mass planet could lie in a stable orbit between 0.05 to $100 \mathrm{AU}$. They further show that no Earthmass planet could exist between 1 and 20 AU. As TTVs are sensitive even to small mass objects, there is the possibility of the existence of a moon around HD80606b. Contrary to this, Barnes \& O'Brien (2002) show that no moon massive enough to create a TTV could exist in a stable orbit around the highly eccentric planet.

With the combination of five different mid-transit timing results, the possible mid-transit time of HD80606b spans over 24 minutes. The large discrepancy between each group's result demonstrates the mid-transit timing issue is unlikely due to an astrophysical event. Furthermore, it is of interest that both this work and Hébrard et al. (2010; both space-based observations) agree within less than $1 \sigma$ difference of an early mid-transit time, while the ground-based results determine a mid-transit time several minutes later. We therefore propose the hypothesis that the difference in mid-transit time derives from systematic errors that depend on the method used to observe HD80606b.

\subsection{Complete Transit Fit Parameters}

We determine two independent sets of parameters for HD80606b, which we will discuss and compare in this section. Figure 2 shows MOST's HD80606b transit light curve with the data unbinned and each point representing $80.40 \mathrm{~s}$ of observation. The large gaps between each cluster of data are the result of Earth passing between MOST and HD80606 (Walker et al.
2003). Unfortunately, two of these blackouts occur during large parts of the transit ingress and egress. In order to minimize the effect of these blackouts on our fit, we hold our limb-darkening coefficients constant during the entire fitting routine.

We further minimize the effects of scatter and blackouts in our data by electing to fit for only four parameters. These four parameters are: planetary radius $\left(R_{p}\right)$, inclination $(i)$, mid-transit time $\left(T_{\text {mid }}\right)$, and the out-of-transit baseline flux. In this section, we will denote our results with either a superscript FH or FW, for Hébrard et al. (2010) or Winn et al. (2009) respectively, based on which of the groups parameters were used (e.g., $R_{p}^{\mathrm{FH}}$ or $R_{p}^{\mathrm{FW}} ; i^{\mathrm{FH}}$ or $i^{\mathrm{FW}}$, etc.).

We perform two separate fits using parameters obtained by Hébrard et al. (2010) and Winn et al. (2009) as fixed constraints. These parameters, which we hold constant while fitting, are listed in Table 2. Our two sets of results (corresponding to the two different sets of fixed parameters) for the planetary radius, inclination, and mid-transit time are listed in Table 3 along with the results of both Hébrard et al. (2010) and Winn et al. (2009) for comparison. We also report the $\chi_{r}^{2}$ of each of our fits. However, the difference between the two $\chi_{r}^{2}$ is minimal. This leads us to conclude that while our results determined by using the Hébrard et al. (2010) constant parameters yield a smaller $\chi_{r}^{2}$, the accuracy of our two fits is statistically comparable. This agreement is demonstrated in Figure 2 as we plot our two best fits against each other. The solid blue line represents our best fit employing Hébrard et al. (2010) constraints while the red dashed line is our best fit using Winn et al. (2009) parameters.

We assume that the $R_{p} / R_{*}$ ratio should be the same for both our fits as the transit depth of our data does not change. We find that our $\left(R_{p} / R_{*}\right)^{\mathrm{FW}}$ and $\left(R_{p} / R_{*}\right)^{\mathrm{FH}}$ measurements are separated by $1.2 \sigma$. Regardless of the difference in our stellar radius constraint, we still achieve statistically similar results. Our $R_{p} / R_{*}$ results straddle Hébrard et al. (2010) result. We also find that our $\left(R_{p} / R_{*}\right)^{\mathrm{FW}}$ is the smallest ratio of the set of results whereas Winn et al. (2009) determined the largest $R_{p} / R_{*}$ for HD80606b with a difference of $3 \sigma$. 
The orbital inclination is directly dependent on the stellar radius, making it difficult to compare our two $i^{\mathrm{FH}}$ and $i^{\mathrm{FW}}$ results. We find it interesting that both our $i^{\mathrm{FH}}$ and $i^{\mathrm{FW}}$ results are exactly $1 \sigma$ larger than the inclinations determined by Hébrard et al. (2010) and Winn et al. (2009), respectively. This is likely due from the blackouts occurring in the ingress and egress of our data, which cause a difficulty in fitting the exact curvature of the transit. We do not report an independent estimation of the impact parameter, as we do not fit for this variable. Instead, we calculate $b$ using Equation (1) to allow for comparison and find that our $b^{\mathrm{FH}}$ is slightly larger than our $b^{\mathrm{FW}}$ but less than $1 \sigma$ difference.

Our mid-transit times in both of these complete fits differ from the timing only fits listed in Table 1 because they are the best-fit times based on the other fitted parameters. As we fit for multiple variables, the resulting errors on these times are higher than the ones listed above. We still find a mid-transit time earlier than predicted; however, the spread between our $T_{\mathrm{mid}}^{\mathrm{FW}}$ and $T_{\mathrm{mid}}^{\mathrm{FH}}$ results is a three minute difference, even though they have a less than $1 \sigma$ discrepancy. Interestingly, our $T_{\mathrm{mid}}^{\mathrm{FW}}$ is the earliest of all our mid-transit times, including those reported in Section 4.1, leading us to believe the mid-transit time dependence on the other Winn et al. (2009) parameters is not causing the TTV. Both of our $T_{\text {mid }}^{\mathrm{FW}}$ and $T_{\mathrm{mid}}^{\mathrm{FH}}$ results are within $1 \sigma$ of Hébrard et al. (2010) mid-transit time and are at least $1.2 \sigma$ away from Winn et al. (2009).

We verify our mid-transit time by fitting public spectroscopic data from Hébrard et al. (2010) and our photometric data simultaneously. While the quality of our transit leads to large uncertainties in most of our parameters, we determine a midtransit with less than $1 \sigma$ difference to our $T_{\mathrm{mid}}^{\mathrm{FW}}$. We further confirm the period, eccentricity, and longitude of periapsis determined by Hébrard et al. (2010). Since these do not deviate from the orbital parameters established by Winn et al. (2009), we do not consider it necessary to delve in to this finding. We also fit for the impact parameter and $R_{p} / R_{*}$. However, both values as well as the uncertainties associated with them are extremely skewed. We therefore conclude that fitting our transit for more than four parameters leads to large inaccuracies in our model and only report those parameters fitted with the constant constraints from Winn et al. (2009) and Hébrard et al. (2010).

\section{CONCLUSIONS}

Employing the optical telescope MOST we observe the 2010 January transit of HD80606b. Using the fitting routine detailed in Barnes \& Fortney (2003) and modifying it for the high eccentricity of this planet's orbit, we address two separate goals. We first investigate a mid-transit timing discrepancy discovered by Hébrard et al. (2010) and find that our mid-transit time of $2455210.6449 \pm 0.0034$ HJD is within $1 \sigma$ of Hébrard et al. (2010) results and 20 minutes earlier ( $3 \sigma$ difference) than the predicted value determined by Winn et al. (2009).

As Wu \& Murray (2003) have theoretically ruled out the possibility for a separate planet causing these variations and
Barnes \& O'Brien (2002) discarding the hypothesis for the existence of an exomoon, we think the TTV is caused by systematic errors depending on the contrasting methods of ground-based and space-based observations.

We also present an independent set of parameters for the planetary radius, orbital inclination, and mid-transit time of HD80606b derived from the MOST photometry. We use the orbital and stellar parameters determined by Winn et al. (2009) and Hébrard et al. (2010) and hold them constant during the fitting process. We find that our two sets of results are statistically similar to each other and there are no unusual discrepancies occurring even with different constraints. With Hébrard et al. (2010) parameters, we further determine that all of our results are within $1 \sigma$ of their results. However, we also discover that our fitted planetary radius is $2.2 \sigma$ smaller than Winn et al. (2009) result.

When fitting for our own independent mid-transit time, we find that our mid-transit times differ between each other by three minutes but remain less than $1 \sigma$ apart. Furthermore they are 18 minutes earlier than predicted by Winn et al. (2009). We also conclude that the mid-transit timing issue is not due to the differing in the orbital or stellar parameters of Winn et al. (2009) compared to Hébrard et al. (2010).

As we are currently relying on the observations of only two transits, we lack the data necessary to draw any sound conclusions regarding the discrepancies in our results. Future observations, both with ground-based and space-based telescopes, are a necessity in order to better address the transit timing issue. These observations will also further constrain the orbital, stellar, and planetary parameters of HD80606b and aid in bettering our understanding of the formation and evolution of this unique system.

We acknowledge funding from the NASA MOST Guest Observer Program, grant No. NNX10AI84G.

\section{REFERENCES}

Barnes, J. W. 2007, PASP, 119, 986

Barnes, J. W., \& Fortney, J. J. 2003, ApJ, 588, 545

Barnes, J. W., \& O’Brien, D. P. 2002, ApJ, 575, 1087

Brown, T. M., Charbonneau, D., Gilliland, R. L., Noyes, R. W., \& Burrows, A. 2001, ApJ, 552, 699

Fossey, S. J., Waldmann, I. P., \& Kipping, D. M. 2009, MNRAS, 396, L16

Garcia-Melendo, E., \& McCullough, P. R. 2009, ApJ, 698, 558

Hébrard, G., Dsert, J.-M., Daz, R. F., et al. 2010, A\&A, 516, A95

Hidas, M. G., Tsapras, Y., Mislis, D., et al. 2010, MNRAS, 406, 1146

Laughlin, G., Deming, D., Langton, J., et al. 2009, Natur, 457, 562

Moutou, C., Hébrard, G., Bouchy, F., et al. 2009, A\&A, 498, L5

Naef, D., Latham, D. W., Mayor, M., et al. 2001, A\&A, 375, L27

Pont, F., Hebrard, G., Irwin, J. M., et al. 2009, A\&A, 502, 695

Press, W. H., Teukolsky, S. A., Vetterling, W. T., \& Flannery, B. P. 1992, Numerical Recipes in C. The Art of Scientific Computing (Cambridge: Univ. Press)

Rowe, J. F., Matthews, J. M., Seager, S., et al. 2006, ApJ, 646, 1241 Shporer, A., Winn, J. N., Dreizler, S., et al. 2010, ApJ, 722, 880

Walker, G., Matthews, J., Kuschnig, R., et al. 2003, PASP, 115, 1023

Winn, J. N., Howard, A. W., Johnson, J. A., et al. 2009, ApJ, 703, 2091

Wu, Y., \& Murray, N. 2003, ApJ, 589, 605 